This is an Open Access article distributed under the terms of the Creative Commons Attribution-Noncommercial License, which permits unrestricted use, distribution, and reproduction in any noncommercial medium, provided the original work is properly cited.

\title{
SPECTRAL LINE CONFUSION IN SAFARI SURVEYS (WORK IN PROGRESS)
}

\author{
G. Raymond and K. G. Isaak \\ Cardiff University, School of Physics \& Astronomy, Queens Buildings, The Parade, Cardiff, CF24 3AA, UK
}

\begin{abstract}
Source confusion occurs when sources are clustered to the order of the telescope beam size on the sky, and determines the useful depth to which to take large-area extragalactic surveys (cf. discussion in Raymond et al. these proceedings). Line, or spectral confusion occurs when multiple sources are observed by a spectrometer in a single telescope beam: the spectra from two or more objects are effectively scrambled.

In this poster we present the preliminary results of an investigation into how well redshifts can be estimated from deep spectroscopic surveys with SAFARI-type observations in which more than one source is observed in a single, spatial element.
\end{abstract}

Key words: Galaxies: formation - Missions: SPICA

\section{INTRODUCTION}

Source confusion may be defined as the degradation of the quality of photometry of sources projected on a scale to the order of the telescope beam size (Scheuer, 1957). The confusion limit sets the useful depth to which large-area extra-galactic surveys should be taken. For example, the Herschel mission (Pilbratt, 2004) (launched in 2009) has a $3.5 \mathrm{~m}$ diameter mirror with a resultant angular resolution of $\sim 8$ " at $120 \mu \mathrm{m}$. At these wavelengths, the confusion limit for such a mirror is estimated to be around $\sim 5 \mathrm{mJy}$ (Dole et al., 2004; Jeong et al., 2006; Le Borgne et al., 2009). Surveys at $24 \mu \mathrm{m}$ suggest that at these flux levels one will only be able to resolve at most $\sim 50 \%$ of the cosmic infrared background (CIB) (Dole et al., 2004; Le Borgne et al., 2009). It would be possible to reduce the confusion limit at a given wavelength by moving to a larger diameter mirror. Far-infrared (FIR) observations, however, are necessarily made from space, which places a practical limit on the telescope diameter and thus by how much we can reduce the confusion limit.

One way to break the confusion limit is to make use of the extra dimension of wavelength, to which one has access in spectroscopic surveys. Discrete sources can be identified by relatively bright emission lines: by comparing rest-wavelengths with observed wavelengths one can uniquely determine redshift and thus identify sources.
Work presented in Clements et al. (2007), Raymond et al. (2009) and Raymond et al. these proceedings, modeled artificial skies matched to the instrumental parameters of SAFARI, the proposed FIR imaging spectrometer for SPICA (Swinyard et al., 2006). These were populated with sources based on the evolutionary models of Pearson (2005) and Pearson et al. (2007). Clements et al. implemented a manual redshift determination method on a single SAFARI field of view (FoV) using FIR emission lines only. Raymond et al. extended on this by using both FIR and mid-infrared (MIR) emission lines and through implementing an automated redshift estimation method. Using these methods it was found it was possible to estimate spectroscopic redshifts for sources up to an order of magnitude below the traditional $120 \mu \mathrm{m}$ continuum confusion limit.

The use of blind spectral line surveys to resolve FIR sources is not without its own limitations and type of confusion. Line or spectral confusion occurs when more than one source is found in a single telescope beam: the spectra from the different objects are effectively scrambled, and it can become difficult to determine which lines are emitted by which object. As a result, source redshifts become hard to extract. Raymond et al. (2009) attempted to extract redshifts from spectrally confused spectra, however no systematic analysis was performed on estimating redshifts from confused spectra. In this work we rectify this, and present the preliminary results of a systematic analysis of our ability to extract redshifts from scrambled spectra.

\section{MethoD}

The spectra used in this work are a sub-sample of those used in Raymond et al. (2009) and are shown in Figure 1. We selected the following 6 spectra to represent each of the principle classes of object; normal (eg. NGC 7331); star forming - starburst (eg. M82); luminous/ultraluminous cold LIRG (eg. NGC 253) and cold ULIRG (eg. Arp220); Seyfert - Seyfert 1 (eg. Mrk 1014) and Seyfert 2 (eg. NGC 1068). These spectra are highlighted in Figure 1 by a thicker line. Details as to how the spectra were compiled are given Raymond et al. (2009).

We generate sets of combined spectra by taking every pairwise combination of the spectral classes listed above, normalized to luminosities of $L_{I R}=10^{10} L_{\odot}, 10^{11} L_{\odot}, 10^{12} L_{\odot}$ 


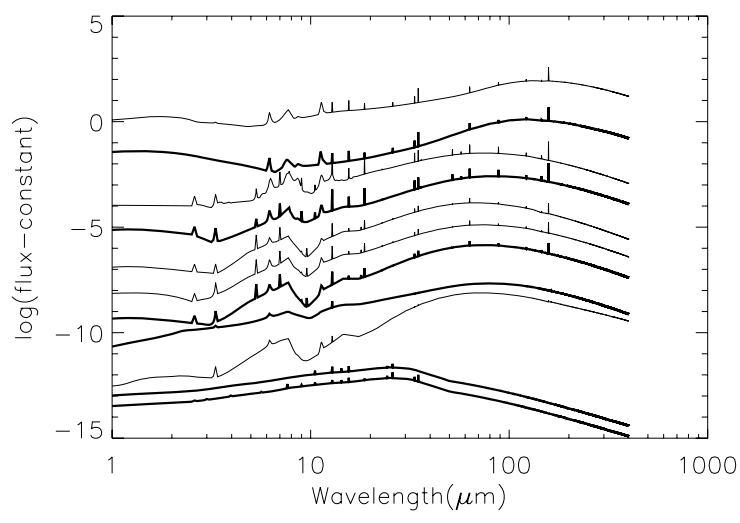

Figure 1. Spectra used in this work and in Raymond et al. (2009). From the top downwards; normal cold, normal (eg. NGC 7331), starburst, M82 starburst (eg. M82), $10^{11} L_{\odot}$ LIRG, $10^{11.5} L_{\odot}$ LIRG, cold LIRG (eg. NGC 253), hot ULIRG, cold ULIRG (eg. Arp220), Sey 1 (eg. Mrk 1014) and Sey 2 (eg. NGC 1068). Only spectra highlighted in bold have been used in this study.

and redshifted in steps of $\Delta z=0.2$, between $0.2<z<4$. We approximate the resolution of SAFARI as a wavelengthindependent value of $R \sim 1000$. Gaussian noise at level commensurate with 10 hours of integration with SAFARI, $\sigma=0.3 \mathrm{mJy}$, is then added to the combined spectrum. We then attempt to estimate the redshift of one or both of these sources.

We estimate redshifts using the pseudo-cross-correlation method of Raymond et al. (2009) and also Raymond et al. these proceedings. This automated redshift estimation method first fits a fourth order polynomial to the continuum of the combined spectrum. This fit is then subtracted from the combined spectrum leaving an array containing only emission lines and noise. The highest flux pixel in this array is considered to be real (rather than noise) and an array of possible redshifts is generated assuming the wavelength to be each of the lines in our line template set. The set of template lines is then placed at each of these redshifts and the redshift which gives the best correlation between our continuum-subtracted spectrum and the shifted template lines is considered to be the correct redshift. This process is then repeated on any remaining, unassigned, potential emission lines, in order to estimate the redshift of the second source.

\section{Results \& Discussion}

It can be seen from Figure 2 that we are able to accurately ${ }^{1}$ estimate at least one redshift from a combined spectrum, provided that the combined $120 \mu \mathrm{m}$ flux, $S_{120 \mu m}>0.3 \mathrm{mJy}$.

\footnotetext{
1 An estimated source redshift is defined as being accurate if it differs from the input redshift by less than 0.1 , i.e. $\left|z_{\text {evaluated }}-z_{\text {catalog }}\right|=\Delta z<0.1$
}

We are also able to accurately estimate both redshifts over a wide range of individual source fluxes. The most significant factor that determines the efficiency of our accurate redshift estimation is the flux of the sources. As can be seen in Figure 3, our accurate redshift estimation efficiency depends, unsurprisingly, both on the ratio of the $120 \mu \mathrm{m}$ fluxes as well the flux of each individual source. The accurate redshift estimation efficiency is highest when both spectra are bright. Figure 2 shows that both redshifts are accurately estimated most often when the $120 \mu \mathrm{m}$ flux ratio $S_{120 \mu m}^{1} / S_{120 \mu m}^{2} \gtrsim 10$, however from Figure 3 we see that both redshifts can be accurately estimated for combined spectra with an even larger flux ratio than this if both sources have high $120 \mu \mathrm{m}$ fluxes.

The accurate redshift estimation rates are also highly dependent on spectral type: this can be seen in Figure 4 which shows the spectral combinations which give the highest and lowest rates of accurate redshift estimation. Again, this is unsurprising given the variation in line strength with source type. In the case of cold LIRG/cold LIRG combination, there are a large number of strong emission lines, therefore we are able to accurately estimate redshifts over a wide range of input redshifts. In the case of the cold ULIRG/ cold ULIRG spectral combination, however, the scrambled spectrum contains only a small number of very weak emission lines, therefore the accurate redshift estimation efficiency is very low.

\section{Conclusions}

The most important factors affecting the efficiency of our accurate redshift estimation are the $120 \mu \mathrm{m}$ fluxes and the ratio of the $120 \mu \mathrm{m}$ fluxes of the two sources making up the combined spectrum. We find that accurate redshift estimation is most efficient when the ratio of the $120 \mu \mathrm{m}$ fluxes, $S_{120 \mu m}^{1} / S_{120 \mu m}^{2} \gtrsim 10$, and the brighter the component sources, the easier it is to accurately estimate their redshifts.

The efficiency of accurate redshift estimation also depends strongly on spectral type, as on average it is easier to estimate redshifts for sources with a high number of strong emission lines. The effect of spectral type on accurate redshift estimation efficiency will be the subject of further investigation, as there may be a strong dependence on particular emission lines appearing in the SAFARI waveband over certain redshift regimes.

In Figure 5, on average the efficiency of accurate estimation of redshifts drops with increasing redshift and therefore decreasing flux. The relationship between redshift estimation and redshift is more complicated than this however, as for particular spectral type/redshift combinations, emission line degeneracies can occur. This can cause good fits to be found for inaccurate redshifts. Figure 5 does not have as smooth a drop off with increasing redshift (and therefore decreasing flux) as the flux vs flux plot in Figure 3, and this is likely due to the aforementioned 

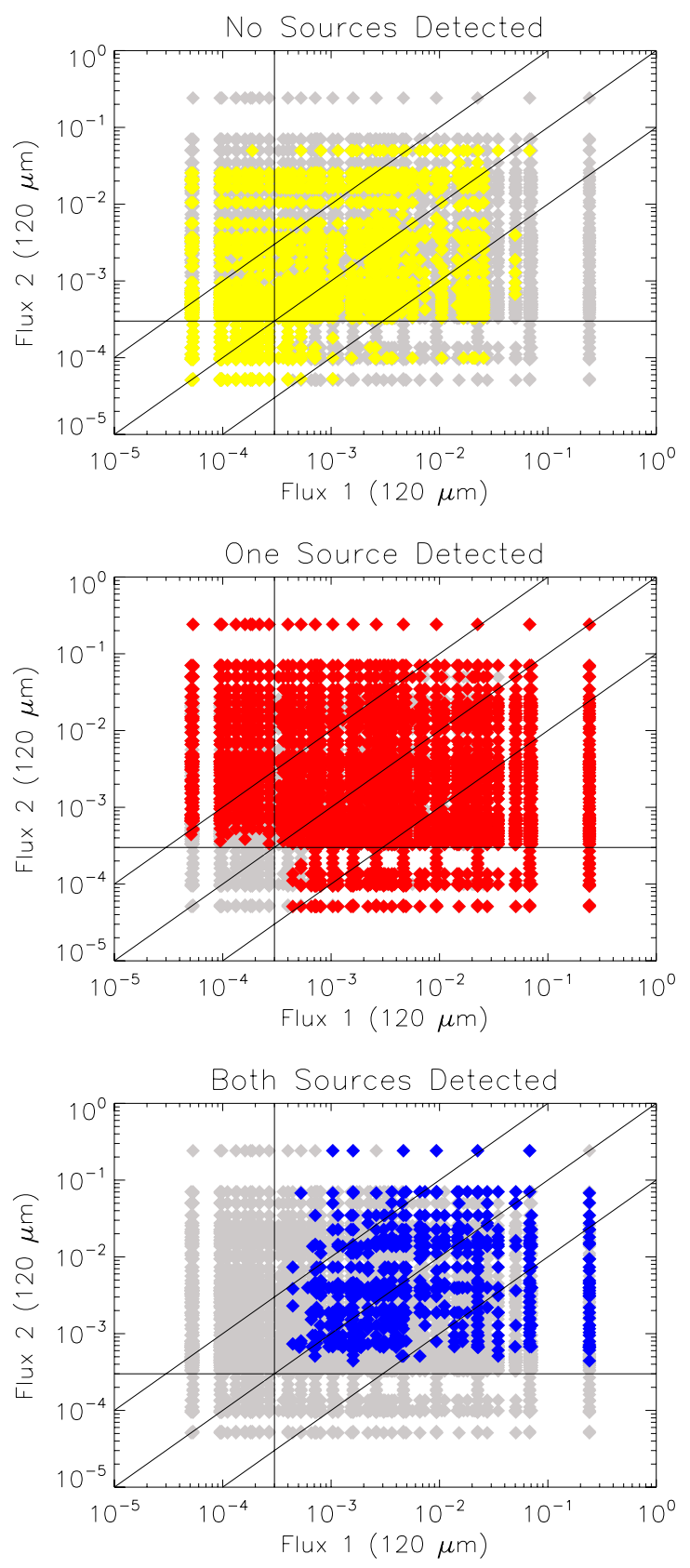

Figure 2. The $S_{120 \mu m}$ vs $S_{120 \mu m}$ distribution for each combination of spectral types over the redshift range from $0 \leq z \leq 4$ The grey dots represent all source combinations investigated, the blue where we accurately estimate both redshifts, the red where we accurately estimate one redshift and yellow where we are able to accurately estimate none. We can estimate at least one redshift accurately from combined spectra, provided the combined $120 \mu \mathrm{m}$ flux, $S_{120 \mu m}>0.3 \mathrm{mJy}$. We are able to estimate both redshifts over a wide range of individual fluxes, however the accurate estimation of both redshifts seems to be easiest when the ratio of the individual $120 \mu \mathrm{m}$ fluxes, $S_{120 \mu m}^{1} / S_{120 \mu m}^{2}>10$. Missing grey dots are due to the excising of degenerate spectral combinations. The vertical/horizontal line denote the $0.3 \mathrm{mJy}$ level, and the diagonal lines the flux ratios of 1 and 10 .

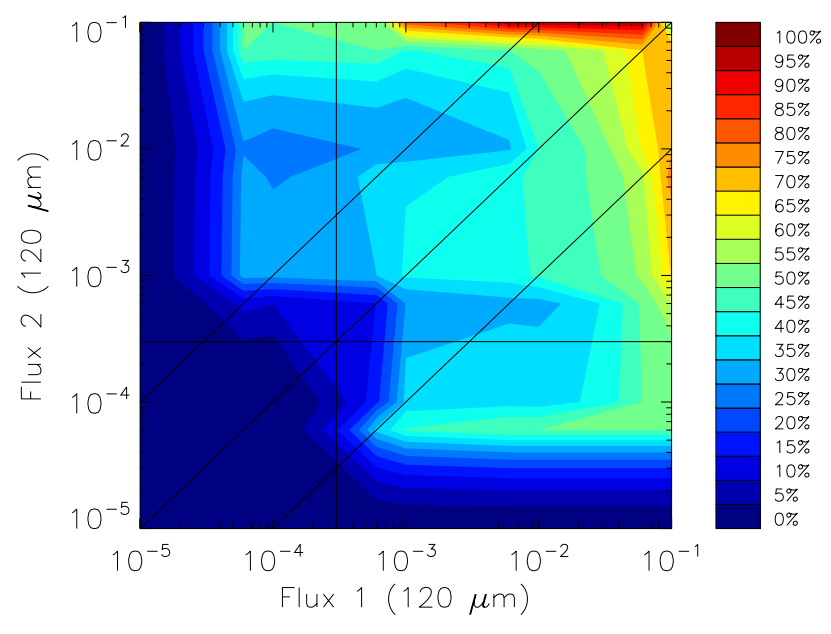

Figure 3. A contour map representing the average percentage of possible sources with accurately estimated redshifts (the maximum number being 2) at a given $S_{120 \mu m}^{1}$ and $S_{120 \mu m}^{2}$, for all combinations of spectra. This shows the same relationship as seen in figure 2, however we now combine the 3 plots into a single plot. Again, the efficiency of our accurate redshift estimation depends both on the flux of each individual source as well as the ratio of the fluxes of the sources. On average it is easiest to accurately estimate redshifts for both of the sources when $S_{120 \mu m}^{1} / S_{120 \mu m}^{2}>10$. If the flux of each component source is high, however, it is possible to accurately estimate redshifts for both sources even when the ratio of their individual fluxes is greater than ten.

effects. The systematic analysis of these more complicated dependencies will be the subject of further investigation.

This work presents the results of a very preliminary analysis. A more detailed analysis of all the issues discussed here is currently underway. We are also considering the recovery of redshifts and line fluxes using emission line templates based on Veilleux et al. (2009) (as noted in Isaak et al. these proceedings).

\section{REFERENCES}

Clements, D. L., Isaak, K. G., Madden, S. C., Pearson, C. 2007, A\&A, 465, 125

Dole, H., et al. 2004, ApJS, 154, 93

Jeong, W. -S., Pearson, C. P., Lee, H. M., Pak, S. J., Nakagawa, T. 2006, MNRAS, 369, 281

Le Borgne, D., Elbaz, D., Ocvirk, P., Pichon. C 2009, A\&A, 504, 727

Pearson, C. P. 2005, MNRAS, 358, 1417

Pearson, C. P., Jeong, W.-S., Lee, H. M., Nakagawa, T. 2007, Advances in Space Research, 40, 605

Pilbratt, G. L. 2004, Presented at the Society of PhotoOptical Instrumentation Engineers (SPIE) Conference, 5481, 401

Raymond, G., Isaak, K. G., Clements, D. L., Rykala, A., Pearson, C. 2009, submitted 

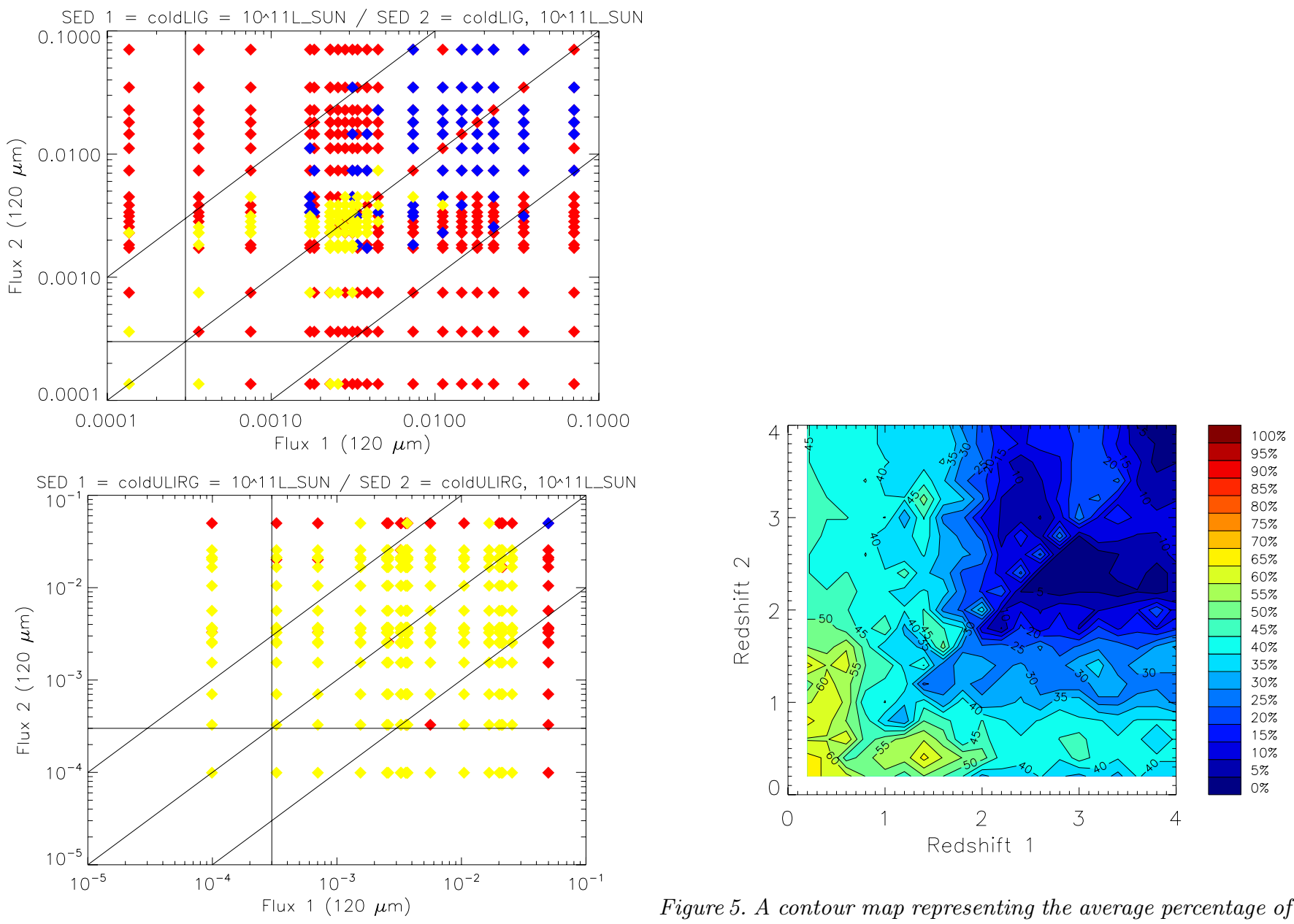

Figure 4. The $S_{120 \mu m}$ vs $S_{120 \mu m}$ distribution for the cold LIRG/cold LIRG (upper panel) and cold ULIRG/cold ULIRG (lower panel) spectral combinations which respectively represent our highest and lowest accurate redshift estimation rates. In the case of the cold LIRG/cold LIRG spectral combination there are a large number of strong emission lines, and we are therefore able to accurately estimate redshifts over a wide range of input redshifts. In contrast, in the case of the cold ULIRG/ cold ULIRG spectral combination, the spectra contain a small number of very weak emission lines, and the redshift estimation efficiency is correspondingly low.

Scheuer, P. A. G. 1957, Proceedings of the Cambridge

Philisophical Society, 53, 764

Swinyard, B., et al. 2006, Proc SPIE, in press

Veilleux, S., et al. 2009, ApJS, 182, 628

Figure 5. A contour map representing the average percentage of possible sources with accurately estimated redshifts (the maximum number being 2) at a given $z_{1}$ and $z_{2}$, for all combinations of spectra (at a fixed luminosity of $10^{11} L_{F I R}$ ). Unsurprisingly, on average the efficiency of accurate estimation of redshifts drops with increasing redshift and therefore decreasing flux. The relationship between redshift estimation and redshift is more complicated than this however, as for particular spectral type/redshift combinations, emission line degeneracies can occur. This can cause good fits to be found for inaccurate redshifts. In addition, there may be a strong dependence on particular emission lines appearing in the SAFARI waveband over certain redshift regimes. The systematic analysis of these more complicated dependencies will be the subject of further investigation. 